\title{
Phylogeny of Some Melanoleuca Species (Fungi: Basidiomycota) in Turkey and Identification of Melanoleuca angelesiana A.H. Sm. As a First Record
}

\author{
Aysenur KALMER ${ }^{1}$, İsmail ACAR ${ }^{2}$, Ayten DIZKIRICI TEKPINAR ${ }^{1 *}$
}

\begin{abstract}
${ }^{1}$ Department of Molecular Biology and Genetics, Van Yüzüncü Y1l University, 65080, Van, Turkey
${ }^{2}$ Department of Organic Agriculture, Başkale Vocational High School, Van Yüzüncü Y1l University, 65080, Van, Turkey

*Corresponding author: aytendizkirici@gmail.com
\end{abstract}

\begin{abstract}
Aim of Study: The purposes of the present study are to describe and identify all collected Melanoleuca samples using morphological characters, determine phylogenetic relationships among species using DNA sequences of ITS and LSU regions and check the number of subgenera of Melanoleuca genus.

Area of Study: Samples were collected from different parts of Turkey and the study was conducted at the Department of Molecular Biology and Genetics in Van Yüzüncü Yll University.

Material and Methods: Twenty-two samples, representatives of fourteen species, were used. Structures of pileus, stipe, lamellae and basidia, cystidia, spores were used as macroscopic and microscopic features, respectively. The nuclear ribosomal internal transcribed spacer region (nrITS) and largest subunit (LSU) were amplified for molecular analyses.

Main results: Taxonomic positions of some Melanoleuca species from Turkey were evaluated based on both morphological characters and sequences of two different nuclear DNA regions. nrITS region was more informative (112 variable sites in ITS1, 1 in $5.8 \mathrm{~S}$ and 95 in ITS2 subregions) compared to LSU (46 variable sites). Analyses of ITS region revealed that the genus Melanoleuca was monophyletic and divided into two subgenera as Melanoleuca and Urticocystis.

Research highlights: Within studied species, Melanoleuca angelesiana was firstly reported for mycobiota of Turkey

Keywords: ITS, LSU, Melanoleuca, Fungal taxonomy, New record
\end{abstract}

\section{Türkiye’deki Bazı Melanoleuca türlerinin (Fungi: Basidiomycota)}

\section{Filogenisi ve Yeni bir Kayıt Olarak Melanoleuca angelesiana A.H. Sm.}

\section{Türünün Tanımlanması}

Öz

Çalış̧manın Amacı: Bu çalışmanın amacı toplanan Melanoleuca örneklerinin morfolojik karakterlerle tanımlanması, ITS ve LSU DNA sekanslarını kullanarak türlerin filogenetik ilişkilerinin belirlenmesi ve Melanoleuca cinsinin alt cins sayısının teyit edilmesidir.

Çalışma Alanı: Örnekler Türkiye'nin farklı bölgelerinden toplanmış ve çalışma Van Yüzüncü Yıl Üniversitesi Moleküler Biyoloji ve Genetik Bölümü'nde gerçekleştirilmiştir.

Material ve Yöntemler: On dört türü temsil eden yirmi iki örnek kullanılmışır. Şapka, sap, lamel yapıları ve bazidya, sistidya, spor yapıları sırasıyla makroskopik ve mikroskopik özellikler olarak kullanılmıştır. Nükleer ribozomal iç aralayıcı bölge (nrITS) ve ribosomal en büyük alt birim (LSU) moleküler analizler için amplifiye edilmiştir.

Ana sonuçlar: Bu çalışmada, Türkiye'den bazı Melanoleuca türlerinin taksonomik konumları hem morfolojik karakterleri hemde iki farklı nükleer DNA bölgesinin sekansları ile değerlendirilmiştir. nrITS bölgesi (ITS1'de 112, 5,8S'de 1, ve ITS2'de 95), LSU bölgesine (46 değişken alan) kiyasla daha bilgilendirici olmuştur. ITS bölgesinin analizleri Melanoluca cinsinin monofletik olduğunu ve Melanoleuca ve Urticocystis olarak iki alt cinse ayrıldığını göstermiştir.

Araştırma konulart: İncelenen türler içerisinde Melanoleuca angelesiana Türkiye mikobiyotası için ilk defa rapor edilmiştir.

Anahtar kelimeler: ITS, LSU, Melanoleuca, Fungal taksonomi, Yeni kayıt 


\section{Introduction}

Melanoleuca Pat. (Tricholomataceae R. Heim ex Pouzar) is a mostly edible saprotrophic fungal genus growing on soil in grasslands, evergreen forests and sand dunes (Boekhout, 1988; Phillips, 2006). Edible fungi have economic and ecologic importance (Yıldız, Gürgen \& Can, 2017). The database of Mycobank lists 333 Melanoleuca species worldwide (http://www.mycobank.org, accessed; 25.02.2018) and only 23 of them have been identified up to now in Turkey (Sesli and Denchev, 2014; Solak, Işıloğlu, Erbil \& Allı, 2015; Acar, Dizkirici Tekpınar, Kalmer \& Uzun, 2017).

The genus is characterized by: collybioid to tricholomatoid basidiomata; emarginate to adnate to subdecurrent lamellae; a white to pale yellowish spore print; hyaline spores with ellipsoid, mostly amyloid ornamentation; absent or present cheilocystidia of two types; pleurocystidia similar to cheilocystidia; pileipellis a cutis to a trichoderm and an absence of clamp connections (Singer, 1986; Bon, 1991; Boekhout, 1999). Especially, structure of the cheilocystidia is a valuable character to identify taxonomic position of the studied species within the genus. Furthermore, the size, amount and ornamentation of spores are important features for delimitation of species (Kibby, 2016). The genus Melanoleuca can be distinguished from other genera with specific shapes of cystidia (thin walled and fusiform to lageniform). Even though these mentioned characters are useful to identify a sample at the genus level, lots of problems are encountered during identification of a sample at the species level because of morphological variabilities and macroscopic/microscopic similarities (Vizzini, Para, Fontenla, Ghignone \& Ercole, 2011; Duriska, Antonin, Para, Tomsovsky \& Jancovicova, 2017).

Some researchers divided Melanoleuca genus into three subgenera as Acystis, Urticocystis and Melanoleuca, using traditional classification which is based on presence and shape of cystidia (Bon 1991). Acystis is characterized by missing cystidia, Urticocystis with urticiform cystidia and Melanoleuca with macrocystidia (Bon, 1991; Bas, Kuyper, Noordeloos \& Vellinga, 1999). However, the infrageneric classifications of the genus have always been unclear ( $\mathrm{Yu}$ et al., 2014) since some morphological and ecological features can be changed due to geographic distribution. Interpretations of morphological characters often varied among mycologists and resulted in disagreements (Hyde et al., 2013). The molecular data may provide invaluable information to identify macrofungus correctly (Undan et al., 2016). Therefore, researchers have begun to use molecular and morphological data together. Vizzini et al. (2011) used sequence of $n r D N A$ ITS region to check the traditional Melanoleuca classification. Their results indicated that Melanoleuca is a monophyletic genus including only two subgenera, Urticocystis and Melanoleuca.

In the present study, specimens were collected from different locations of Turkey and identified by using morphological characters. DNA sequences of ITS (Internal Transcribed Spacer) and LSU (Large Subunit of rRNA) regions were analyzed to understand phylogenetic relationships among Melanoleuca species. These two regions were selected for the study because rDNA gene has been conserved during evolutionary process and usefulness of ITS and LSU regions has been proved by several studies for the Melanoleuca genus (Hinrikson, Hurst, Lott, Warnock \& Morrison, 2005; Brown, Rigdon-Huss, \& Jumpponen, 2014). Especially, ITS region is accepted as a valuable DNA marker for fungal taxonomy (Schoch et al. 2012).

The purposes of the present study are to i) describe and identify all collected samples using morphological characters ii) determine phylogenetic relationships among species using DNA sequences of ITS and LSU regions iii) check the number of subgenera.

\section{Materials and methods \\ Taxon sampling and morphological studies}

The macrofungus samples were collected from different locations of Turkey (Table 1). Twenty-two samples, representatives of 14 species, were collected and used for morphological and molecular studies. All collected samples were deposited in the Fungarium of Van Yüzüncü Yıl University (VANF). Specimens were photographed in situ, using with a Canon (EOS 60D) camera equipped with Tokina $100 \mathrm{~mm}$ macro lens 
during field work. Macroscopic characters were recorded using fresh materials. Pilea, cheilocystidia and basidia were observed in distilled water under a Leica EZ4 stereo microscope and sections were examined under a Leica DM500 research microscope. Microscopic characters were measured with the Leica Application Suite (version 3.2.0) program and described based on different studies (Murrill, 1913; Bresinsky and Stangl, 1977; Bon, 1991; Breitenbach and Kränzlin, 1991; Dähncke, 2004; Jordan, 2004; Gerault, 2005; Clémençon 2009; Vizzini et al., 2011; Buczacki, 2012; Garcia, Blanco \& Matheney 2013; Kuo and Methven, 2014; Desjardin, Wood \& Stevens, 2015).

Table 1. List of studied Melanoleuca species, their localities, collected date, subgenera and NCBI accession numbers. Subgenera were decided using study of Vizzini et al. (2011).

\begin{tabular}{|c|c|c|c|c|c|}
\hline \multirow[t]{2}{*}{ Species } & \multirow[t]{2}{*}{ Locality } & \multirow{2}{*}{$\begin{array}{l}\text { Collected } \\
\text { date }\end{array}$} & \multirow[t]{2}{*}{ Subgenera } & \multicolumn{2}{|c|}{ Accession no. } \\
\hline & & & & ITS & LSU \\
\hline \multirow[t]{2}{*}{ 1. M. brevipes (Bull.) Pat. } & Bingöl & 2009 & \multirow[t]{2}{*}{ Urticocystis } & \multirow[t]{2}{*}{ MG989678 } & \multirow[t]{2}{*}{ MG989689 } \\
\hline & Tatvan-Bitlis & $2010-2011$ & & & \\
\hline \multirow[t]{4}{*}{ 2. M. exscissa (Fr.) Singer } & $\begin{array}{c}\text { Hani- } \\
\text { Diyarbakır }\end{array}$ & $\begin{array}{l}2009- \\
2010\end{array}$ & \multirow[t]{4}{*}{ Urticocystis } & \multirow[t]{4}{*}{ MG989683 } & \multirow[t]{4}{*}{ MG989694 } \\
\hline & Erzincan & 2006 & & & \\
\hline & Bingöl & 2009 & & & \\
\hline & Artvin & 2009 & & & \\
\hline $\begin{array}{l}\text { 3. M. graminicola (Velen.) } \\
\text { Kühner \& Maire }\end{array}$ & Baykan-Siirt & 2012 & Urticocystis & MG989684 & MG989695 \\
\hline $\begin{array}{l}\text { 4. M. grammopodia (Bull.) } \\
\text { Murrill }\end{array}$ & $\begin{array}{l}\text { Hani- } \\
\text { Diyarbakır }\end{array}$ & 2010 & Urticocystis & MG989681 & MG989692 \\
\hline \multirow{2}{*}{$\begin{array}{l}\text { 5. M. paedida (Fr.) Kühner \& } \\
\text { Maire }\end{array}$} & Bingöl & 2009 & \multirow{4}{*}{$\begin{array}{l}\text { Urticocystis } \\
\text { Urticocystis }\end{array}$} & \multirow{4}{*}{$\begin{array}{l}\text { MG989679 } \\
\text { MG989680 }\end{array}$} & \multirow{4}{*}{$\begin{array}{l}\text { MG989690 } \\
\text { MG989691 }\end{array}$} \\
\hline & Tatvan-Bitlis & 2010 & & & \\
\hline \multirow[t]{2}{*}{ 6. M. substrictipes Kühner } & Selim-Kars & 2013 & & & \\
\hline & Tatvan-Bitlis & 2011 & & & \\
\hline 7. M. dryophila Murrill & $\begin{array}{l}\text { Şemdinli- } \\
\text { Hakkari }\end{array}$ & 2014 & Urticocystis & KX507366 & KX507363 \\
\hline $\begin{array}{l}\text { 8. M. microcephala (P. Karst.) } \\
\text { Singer }\end{array}$ & Hakkari & 2014 & Urticocystis & MG989677 & MG989688 \\
\hline 9. M. angelesiana A.H. Sm. & Soğanl1-Kars & 2011 & Urticocystis & MG989682 & MG989693 \\
\hline 10. M. arcuata (Bull.) Singer & Siirt & 2016 & Urticocystis & MG989686 & MG989697 \\
\hline 11. M. melaleuca (Pers.) & Erzincan & 2006 & \multirow[b]{2}{*}{ Melanoleuca } & \multirow[b]{2}{*}{ MG989685 } & \multirow[b]{2}{*}{ MG989696 } \\
\hline Murrill & Bingöl & 2008 & & & \\
\hline 12. M. polioleuca (Fr.) & Hani- & 2009 & \multirow{3}{*}{ Melanoleuca } & \multirow{3}{*}{ MG989687 } & \multirow{3}{*}{ MG989698 } \\
\hline \multirow[t]{2}{*}{ Kühner \& Maire } & Diyarbakır & & & & \\
\hline & Hakkari & 2014 & & & \\
\hline $\begin{array}{l}\text { 13. M. heterocystidiosa } \\
\text { (Beller \& Bon) Bon }\end{array}$ & $\begin{array}{l}\text { Yüksekova - } \\
\text { Hakkari }\end{array}$ & 2014 & Melanoleuca & KX507368 & KX507365 \\
\hline $\begin{array}{l}\text { 14. M. communis M. Sánchez- } \\
\text { García\&Cifuentes }\end{array}$ & Hakkari & 2015 & Melanoleuca & KX507367 & KX507364 \\
\hline
\end{tabular}

Molecular studies

DNA isolation, PCR amplification and DNA sequencing

Genomic DNA was isolated from fungal material using the CTAB (Cetyltrimethylammonium bromide, PubChem ID 5974) method (Doyle and Doyle, 1987). The purity and quantity of extracted DNA were determined with NanoDrop2000c UV-Vis Spectrophotometer (Thermo Scientific) and $0.8 \%$ agarose gel electrophoresis. Isolated stock DNA was stored at $-20^{\circ} \mathrm{C}$ prior to $\mathrm{PCR}$ amplification.
DNA amplification of each studied region was performed in a $25 \mu \mathrm{l}$ volume mixture containing genomic DNA (10 ng/ $\mu 1), 10 \mathrm{X}$ PCR Buffer, $\mathrm{MgCl}_{2}(25 \mathrm{mM})$, dNTP mixture $(10 \mathrm{mM})$, selected primer pair $(10 \mu \mathrm{M})$, Taq polymerase $(5 \mathrm{u} / \mu \mathrm{l})$ and sterile water.

To amplify ITS (ITS1-5.8S-ITS2) and LSU regions, primer pairs N-nc18S10 5'AGGAGAAGTCGTAACAAG3'/C26A 5'GTTTCTTTTCCTCCGCT3' (Wen et al. 1996) and LROR 5'ACCCGCTGAACT TAAGC3'/LR5 5'TCCTGAGGGAAACTT CG3' (Vilgalys \& Hester 1990) were used, 
respectively. ITS region was amplified under a program consisting of a hot start at $95{ }^{\circ} \mathrm{C}$ for $2 \mathrm{~min}$, followed by 30 cycles of denaturation at $94{ }^{\circ} \mathrm{C}$ for $1 \mathrm{~min}$, annealing at $54^{\circ} \mathrm{C}$ for $1 \mathrm{~min}$, extension at $72{ }^{\circ} \mathrm{C}$ for 2 min, and a final $72^{\circ} \mathrm{C}$ step for $10 \mathrm{~min}$. Same temperature and time conditions were used for amplification of LSU region except annealing $\left(52{ }^{\circ} \mathrm{C}\right)$. PCR products were checked in $1 \%$ TAE agarose gels staining with Gelred dye, and positive reactions were sequenced with forward and reverse PCR primers. Chromatograms were checked for putative reading errors and corrected. The sequences of species were deposited in NCBI database and their accession numbers were given in Table 1.

\section{Sequence alignment and phylogenetic analysis}

BLAST (Altschul, Gish, Miller, Myers \& Lipman,1997) was used to select the most closely related ITS and LSU rDNA sequences from NCBI database (Appendix 1) and all sequences were aligned with the aid of the program ClustalW (Thompson, Higgins \& Gibson, 1994). Alignments were checked and manually adjusted where it was necessary. Totally 40 sequences ( 26 of them retrieved from NCBI) of the genus were included in the phylogenetic analysis of ITS regions. LSU region was less studied compared to ITS so 10 LSU sequences could be downloaded from NCBI and analyzed with our samples. Pluteus cervinus (Schaeff.) P. Kumm. was used as outgroup for testing the monophyly of Melanoleuca (ITS: JX857448, LSU: HM562221, Garcia et al., 2013). Borders of regions were decided considering sequences downloaded [ITS (JX429148, Garcia et al., 2013) and LSU (JX429179, Garcia et al., 2013)].

Phylogenetic trees were constructed using both Maximum Likelihood (ML) and Maximum Parsimony method with aid of Molecular Evolutionary Genetics Analysis software (MEGA 6; Tamura, Stecher, Peterson, Filipski \& Kumar, 2013). TamuraNei model (Tamura and Nei, 1993) and bootstrap analysis with 1000 replications (Felsenstein, 1985) were selected to construct
ML tree. The tree with the highest log likelihood was shown. Initial tree(s) for the heuristic search were obtained automatically by applying Neighbor-Join and BioNJ algorithms to a matrix of pairwise distances estimated using the Maximum Composite Likelihood (MCL) approach, and then, the topology with superior log likelihood value was selected. The Tree-BisectionReconnection (TBR) search method was employed with 100 random addition replications to construct the MP trees and the consensus tree inferred from 10 most parsimonious trees was used. All positions containing gaps and missing data were eliminated.

\section{Results}

Taxonomy

Macroscopic and microscopic characters of studied Melanoleuca species were given in Table 2. Within the studied specimens, Melanoleuca angelesiana A.H. Sm. was reported firstly for Turkey and its characterization was done by using morphological characters as follow.

Specimen examined: Turkey, Kars, Soğanl1, under conifers, $40^{\circ} 24^{\prime} 053^{\prime \prime} \mathrm{K}, 4^{\circ} 29^{\prime} 117^{\prime \prime} \mathrm{D}$, 2525 m, 03.10.2011, Acar, I., 971, VANF. NCBI numbers: MG989682 (ITS region), MG989693 (LSU region).

\section{Description of Melanoleuca angelesiana A.H. Sm.}

Pileus: $60-100 \mathrm{~mm}$ across; convex to young, then flatted, often with a superficial or evident central lump, smooth, damp when fresh, dark brown, discoloration to tan. Lamellae: notched, attached to the stipe, close to cramped, at first greyish-tan, grayish to whitish, sometimes growing lightly pinkish color. Stipe: 30-60 × 5-15 mm, more or less equal, sometimes lightly bulb at the base, fairly smooth, surface fibrillose-striate when maturity, whitish at the apex but brown like the pileus below, basal mycelium white and partial veil absent. Basidia: 23.5-37.2 $\times$ 6.8-10.7 $\mu \mathrm{m}$. Spores: 7-10 $\times 5-6.5 \mu \mathrm{m}$, more or less elliptical, amyloid, finely warts. Pleurocystidia and cheilocystidia absent (Figure 1). 


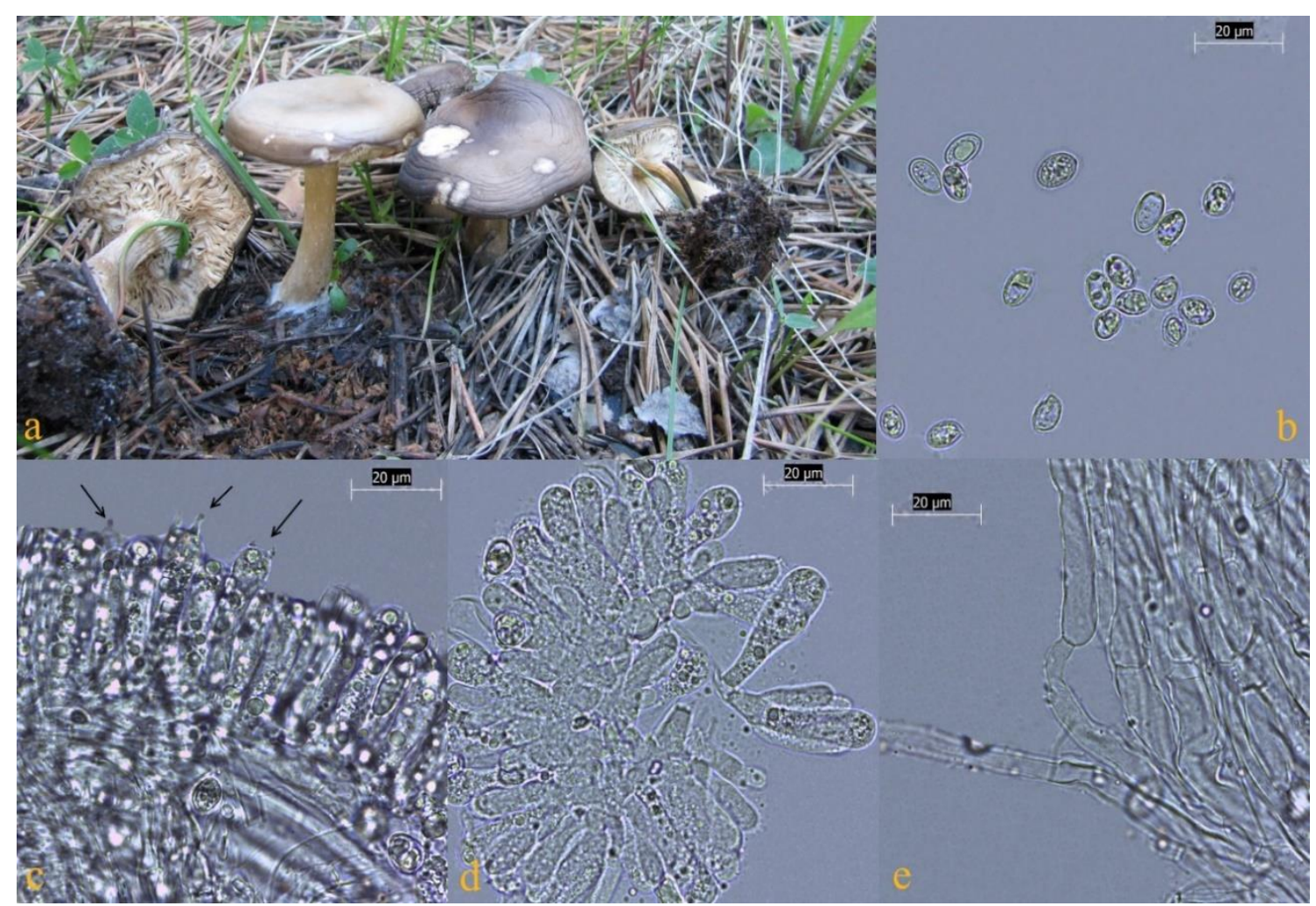

Figure 1. Melanoleuca angelesiana, a) Basidiocarps b) Spores (distilled water) c) Basidia d) Basidioles e) Hyphae

Table 2. Considerable morphologic characters of studied Melanoleuca species

\begin{tabular}{|c|c|c|c|c|c|c|}
\hline Pileus & Lamellae & Stipe & Basidia & Cystidia & Spores & References \\
\hline \multicolumn{7}{|c|}{ M. brevipes (Bull.) Pat. } \\
\hline $\begin{array}{l}50-90 \mathrm{~mm}, \\
\text { convex when } \\
\text { young, then } \\
\text { depressed } \\
\text { center } \\
\text { somewhat } \\
\text { umbonate, } \\
\text { radially } \\
\text { fibrillose, } \\
\text { beige to gray- } \\
\text { brown, grading } \\
\text { to black-brown } \\
\text { toward the } \\
\text { center, }\end{array}$ & $\begin{array}{l}\text { Light cream, later } \\
\text { with gray to } \\
\text { lilactints, slightly } \\
\text { notched to } \\
\text { subdecurent }\end{array}$ & $\begin{array}{l}30-50 \times 8-15 \\
\text { mm, usually } \\
\text { with a clavate } \\
\text { base and } \\
\text { widened apex, } \\
\text { surface with } \\
\text { ocherish on a } \\
\text { brown } \\
\text { background, } \\
\text { covered with } \\
\text { white to } \\
\text { ocherish } \\
\text { powder }\end{array}$ & $\begin{array}{l}30-40 \times \\
8-9 \mu \mathrm{m}, \\
\text { clavate }\end{array}$ & $\begin{array}{l}\text { Cheilocystidia } \\
\text { of the stinging } \\
\text { hair type } \\
\text { urticifom, } \\
\text { Caulocystidia } \\
\text { at the stipe } \\
\text { apex }\end{array}$ & $\begin{array}{l}\text { Elliptic, } \\
\text { irregularly } \\
\text { verrucose, } \\
\text { hyaline } \\
\text { with drops, } \\
6.9-9.7 \times \\
4.6-7.0 \mu \mathrm{m}\end{array}$ & $\begin{array}{l}\text { Boekhout, } \\
\text { 1988; } \\
\text { Breitenbach \& } \\
\text { Kränzlin,1991 }\end{array}$ \\
\hline \multicolumn{7}{|c|}{ M. exscissa (Fr.) Singer } \\
\hline $\begin{array}{l}40-70 \mathrm{~mm}, \\
\text { convex when } \\
\text { young, later } \\
\text { plane and } \\
\text { sometimes } \\
\text { with a } \\
\text { depressed } \\
\text { center, } \\
\text { obtusely } \\
\text { umbonate in } \\
\text { the center }\end{array}$ & $\begin{array}{l}\text { White to cream, } \\
\text { edges slightly } \\
\text { undulating to } \\
\text { crenate }\end{array}$ & $\begin{array}{l}40-60 \times 5-8 \\
\text { mm, } \\
\text { somewhat } \\
\text { enlarged } \\
\text { toward the } \\
\text { apex, surface } \\
\text { fibrillose, } \\
\text { apex white- } \\
\text { pruinose, } \\
\text { dark-brown } \\
\text { toward the } \\
\text { base }\end{array}$ & $\begin{array}{l}22-25 \times \\
6.5-8 \mu \mathrm{m}, \\
\text { cylindric } \\
\text { to } \\
\text { cylindric } \\
\text { clavate }\end{array}$ & $\begin{array}{l}\text { Cheilo- and } \\
\text { pleurocystidia } \\
\text { fusiform to } \\
\text { lageniform }\end{array}$ & $\begin{array}{l}\text { Elliptic, } \\
\text { verrucose, } \\
\text { hyaline, } \\
7.1-8.8 \times \\
4.2-5.5 \mu \mathrm{m}\end{array}$ & $\begin{array}{l}\text { Breitenbach \& } \\
\text { Kränzlin,1991 }\end{array}$ \\
\hline
\end{tabular}


Table 2. (continued)

\begin{tabular}{|c|c|c|c|c|c|c|}
\hline \multicolumn{7}{|c|}{ M. graminicola (Velen.) Kühner \& Maire } \\
\hline $\begin{array}{l}20-30 \mathrm{~mm}, \\
\text { convex- } \\
\text { campanulate } \\
\text { when young, } \\
\text { with a distinct } \\
\text { papilla, later } \\
\text { plan to slightly } \\
\text { depressed, } \\
\text { dark-brown to } \\
\text { dark-gray } \\
\text { brown, center } \\
\text { darker }\end{array}$ & $\begin{array}{l}\text { White, later } \\
\text { cream-whitish, } \\
\text { sometimes also } \\
\text { with a warm pink } \\
\text { tint }\end{array}$ & $\begin{array}{l}40-50 \times 3-4 \\
\text { mm, slightly } \\
\text { clavate toward } \\
\text { the base, } \\
\text { surface cream } \\
\text { to pink- } \\
\text { whitish, with } \\
\text { white fibrils }\end{array}$ & $\begin{array}{l}23-35 \times \\
7-8.5 \mu \mathrm{m} \\
\text { clavate, }\end{array}$ & $\begin{array}{l}\text { Cystidia } \\
\text { absent }\end{array}$ & $\begin{array}{l}\text { Elliptic, } \\
\text { verrucose, } \\
\text { hyaline, } \\
\text { with drops, } \\
5.6-7.9 \times \\
4.6-6.0 \mu \mathrm{m}\end{array}$ & $\begin{array}{l}\text { Breitenbach \& } \\
\text { Kränzlin,1991 }\end{array}$ \\
\hline \multicolumn{7}{|c|}{ M. grammopodia (Bull.) Murrill } \\
\hline $\begin{array}{l}70-150 \mathrm{~mm} \text {, } \\
\text { bell-shaped } \\
\text { then expanded, } \\
\text { often } \\
\text { becoming } \\
\text { centrally } \\
\text { depressed; } \\
\text { light to dark } \\
\text { gray-brown }\end{array}$ & $\begin{array}{l}\text { Crowded sinuate, } \\
\text { rather thick, } \\
\text { triangular- } \\
\text { ventricose, pale } \\
\text { cream-beige }\end{array}$ & $\begin{array}{l}50-120 \times 10- \\
15 \mathrm{~mm}, \\
\text { swollen at the } \\
\text { base; grey- } \\
\text { brown, } \\
\text { longitudinally } \\
\text { fibrillose }\end{array}$ & $\begin{array}{l}30-40 \times \\
8-13 \mu \mathrm{m}, \\
\text { clavate }\end{array}$ & $\begin{array}{l}\text { Cheilocystidia } \\
\text { with swollen } \\
\text { and long, } \\
\text { narrow neck }\end{array}$ & $\begin{array}{l}\text { Elliptical, } \\
\text { minutely } \\
\text { warted, } \\
\text { amyloid, } \\
8.5-9.5 \times 5 \text { - } \\
6 \mu \mathrm{m}\end{array}$ & $\begin{array}{l}\text { Boekhout, } \\
\text { 1988; Phillips, } \\
2006\end{array}$ \\
\hline \multicolumn{7}{|c|}{ M. melaleuca (Pers.) Murrill } \\
\hline $\begin{array}{l}\text { 30-80 mm, } \\
\text { dark-brown, } \\
\text { hygrophanous, } \\
\text { drying buff; at } \\
\text { first flattened } \\
\text { convex, } \\
\text { becoming } \\
\text { umbonate- } \\
\text { depressed }\end{array}$ & $\begin{array}{l}\text { White, } \\
\text { emarginated, } \\
\text { broad, crowded }\end{array}$ & $\begin{array}{l}55-90 \times 5-8 \\
\text { mm, } \\
\text { cylindrical, } \\
\text { slightly } \\
\text { broader } \\
\text { towards base, } \\
\text { sometimes } \\
\text { becoming pale } \\
\text { beige to } \\
\text { brown-grey } \\
\text { longitudinally } \\
\text { fibrillose with } \\
\text { pruinose apex }\end{array}$ & $\begin{array}{l}25-40 \times \\
7-10 \mu \mathrm{m}, \\
\text { clavate }\end{array}$ & $\begin{array}{l}\text { Cystidia } \\
\text { absent }\end{array}$ & $\begin{array}{l}\text { Hyaline, } \\
\text { warty, } \\
\text { ellipsoid, } \\
\text { amyloid, } \\
\text { droplets, 7- } \\
8.5 \times 5-5.5 \\
\mu \mathrm{m}\end{array}$ & $\begin{array}{l}\text { Boekhout, } \\
\text { 1988; Jordan, } \\
2004\end{array}$ \\
\hline \multicolumn{7}{|c|}{ M. paedida (Fr.) Kühner \& Maire } \\
\hline $\begin{array}{l}35-50 \mathrm{~mm}, \\
\text { hemispherical } \\
\text { when young, } \\
\text { later convex to } \\
\text { plane, gray- } \\
\text { brown with a } \\
\text { silver tinge } \\
\text { when dry, } \\
\text { gray-beige } \\
\text { when wet }\end{array}$ & $\begin{array}{l}\text { Gray-whitish to } \\
\text { gray, with } \\
\text { yellowish-brown } \\
\text { edges, }\end{array}$ & $\begin{array}{l}30-50 \times 7-10 \\
\text { mm, cylindric, } \\
\text { some with a } \\
\text { bulbous base } \\
\text { or enlarged } \\
\text { apex, surface } \\
\text { longitudinally } \\
\text { fibrillose, } \\
\text { cream to gray- } \\
\text { brown }\end{array}$ & $\begin{array}{l}27-30 \times \\
6-8 \mu \mathrm{m}, \\
\text { cylindric } \\
\text { to } \\
\text { cylindric- } \\
\text { clavate }\end{array}$ & $\begin{array}{l}\text { Cheilocystidia } \\
40-50 \times 5-8 \\
\mu \mathrm{m}, \text { stinging- } \\
\text { hair shaped, } \\
\text { sometimes } \\
\text { sparse to } \\
\text { absent }\end{array}$ & $\begin{array}{l}8.1-9.6 \times \\
5.5-6.3 \mu \mathrm{m} \\
\text { elliptic, } \\
\text { finely } \\
\text { verrucose, } \\
\text { hyaline }\end{array}$ & $\begin{array}{l}\text { Breitenbach \& } \\
\text { Kränzlin,1991 }\end{array}$ \\
\hline \multicolumn{7}{|c|}{ M. polioleuca (Fr.) Kühner \& Maire } \\
\hline $\begin{array}{l}25-70 \mathrm{~mm} \text {, at } \\
\text { first convex, } \\
\text { finally with } \\
\text { somewhat } \\
\text { depressed } \\
\text { centre, mostly } \\
\text { with low broad } \\
\text { umbo, when } \\
\text { moist } \\
\text { yellowish } \\
\text { brown to dark } \\
\text { grey-brown }\end{array}$ & $\begin{array}{l}\text { Crowded, adnate } \\
\text { to sinuose, } \\
\text { mostly with } \\
\text { subdecurrent } \\
\text { toothlet, } \\
\text { ventricose to } \\
\text { triangular, } \\
\text { whitish }\end{array}$ & $\begin{array}{l}35-85 \times 3-8 \\
\text { mm, } \\
\text { cylindrical } \\
\text { clavate base, } \\
\text { stuffed, at first } \\
\text { whitish, } \\
\text { becoming pale } \\
\text { to sordid grey- } \\
\text { brown }\end{array}$ & $\begin{array}{l}15-40 \times \\
6-10 \mu \mathrm{m}, \\
\text { clavate }\end{array}$ & $\begin{array}{l}\text { Cheilocystidia } \\
45-75 \times 8-15 \\
\mu \mathrm{m}, \text { fusiform } \\
\text { to lageniform, } \\
\text { at apex } \\
\text { encrusted with } \\
\text { crystals }\end{array}$ & $\begin{array}{l}6.3-9 \times 4-5 \\
\mu \mathrm{m} \\
\text { ellipsoid to } \\
\text { elongate, } \\
\text { rather } \\
\text { ornamented }\end{array}$ & Boekhout, 1988 \\
\hline
\end{tabular}


Table 2 (continued)

\begin{tabular}{|c|c|c|c|c|c|c|}
\hline \multicolumn{7}{|c|}{ M. substrictipes Kühner } \\
\hline $\begin{array}{l}25-70 \mathrm{~mm}, \\
\text { convex when } \\
\text { young, later } \\
\text { plane to } \\
\text { irregularly } \\
\text { undulating, } \\
\text { with an } \\
\text { umbonate } \\
\text { center, satiny, } \\
\text { white when } \\
\text { young, later } \\
\text { cream-whitish }\end{array}$ & $\begin{array}{l}\text { White, later } \\
\text { cream colored } \\
\text { with a pink tint }\end{array}$ & $\begin{array}{l}40-70 \times 3-8 \\
\text { mm, } \\
\text { cyclindric, } \\
\text { base bulbous } \\
\text { and up to } 10 \\
\text { mm thick, } \\
\text { sometimes } \\
\text { twisted, } \\
\text { surface } \\
\text { smooth, } \\
\text { longitudinally } \\
\text { fibrillos, white } \\
\text { when young } \\
\text { later yellow- } \\
\text { brownish }\end{array}$ & $\begin{array}{l}33-38 \times \\
7-9 \mu \mathrm{m}, \\
\text { clavate, }\end{array}$ & $\begin{array}{l}\text { Cheilocystidia } \\
\text { fusiform to } \\
\text { subulate, } \\
\text { almost like } \\
\text { stinging hair }\end{array}$ & $\begin{array}{l}8.1-10 \times 5- \\
6.3 \mu \mathrm{m}, \\
\text { elliptic, } \\
\text { finely } \\
\text { verrucose, } \\
\text { hyaline, } \\
\text { with drops }\end{array}$ & $\begin{array}{l}\text { Breitenbach \& } \\
\text { Kränzlin, } 1991\end{array}$ \\
\hline \multicolumn{7}{|c|}{ M. dryophila Murrill } \\
\hline $\begin{array}{l}30-150 \mathrm{~mm}, \\
\text { convex, } \\
\text { gibbous, viscid } \\
\text { when flesh, } \\
\text { subshining, } \\
\text { whitish, } \\
\text { stained with } \\
\text { rusty-brown, } \\
\text { margin paler, } \\
\text { context white, } \\
\text { with } \\
\text { farinaceous }\end{array}$ & $\begin{array}{l}\text { Deeply sinuate to } \\
\text { adnexed, close, } \\
\text { narrow, plane, } \\
\text { white }\end{array}$ & $\begin{array}{l}60-130 \times 10- \\
45 \mathrm{~mm}, \\
\text { stuffed at } \\
\text { maturity, } \\
\text { equal to } \\
\text { enlarged at the } \\
\text { base, fibrillose } \\
\text { brown over a } \\
\text { pallid } \\
\text { background } \\
\text { below }\end{array}$ & & Not seen & $\begin{array}{l}5-7 \times 3.5- \\
4.5 \mu \mathrm{m}, \\
\text { globose, } \\
\text { smooth, } \\
\text { hyaline }\end{array}$ & $\begin{array}{l}\text { Acar et al., } \\
2017\end{array}$ \\
\hline \multicolumn{7}{|c|}{ M. microcephala (P. Karst.) Singer } \\
\hline $\begin{array}{l}10-30 \mathrm{~mm} \text {, low } \\
\text { convex to } \\
\text { plane with a } \\
\text { small umbo, } \\
\text { smooth, yellow } \\
\text { brown to dark } \\
\text { brown }\end{array}$ & $\begin{array}{l}\text { white or tinged } \\
\text { pale buff }\end{array}$ & & & $\begin{array}{l}\text { Cheilocystidia } \\
40-60 \times 6-10 \\
\mu \mathrm{m}, \text { urticoid } \\
\text { septate, often } \\
\text { with crystals at } \\
\text { apex }\end{array}$ & $\begin{array}{l}6-8 \times 4-5 \\
\mu \mathrm{m}, \\
\text { ellipsoid, } \\
\text { verrucose }\end{array}$ & $\begin{array}{l}\text { Vesterholt and } \\
\text { Knudsen, } 2008\end{array}$ \\
\hline \multicolumn{7}{|c|}{ M. heterocystidiosa (Beller \& Bon) Bon } \\
\hline $\begin{array}{l}50-70 \mathrm{~mm} ., \\
\text { convex to } \\
\text { smooth, } \\
\text { slightly } \\
\text { pruinose, gray- } \\
\text { dark brown } \\
\text { with olive in } \\
\text { the center, then } \\
\text { greyish ochre }\end{array}$ & $\begin{array}{l}\text { Somewhat } \\
\text { fragile, closed, } \\
\text { white }\end{array}$ & $\begin{array}{l}50-80 \times 4-7 \\
\text { mm, slightly } \\
\text { pruinose, } \\
\text { grey-sepia, } \\
\text { pale greyish, } \\
\text { downwards } \\
\text { striate, at the } \\
\text { base dark }\end{array}$ & & $\begin{array}{l}\text { Cheilocystidia } \\
35-90 \times 8-20 \\
\mu \mathrm{m}, \text { abundant } \\
\text { and variable in } \\
\text { size and shape, } \\
\text { mostly } \\
\text { vertical- } \\
\text { shaped }\end{array}$ & $\begin{array}{l}7-9 \times 4-5 \\
\mu \mathrm{m}, \\
\text { elliptical, } \\
\text { warty }\end{array}$ & $\begin{array}{l}\text { Acar et al., } \\
2017\end{array}$ \\
\hline \multicolumn{7}{|c|}{ M. communis M. Sánchez-García\&Cifuentes } \\
\hline $\begin{array}{l}30-150 \mathrm{~mm}, \\
\text { plane-convex } \\
\text { to plane, } \\
\text { sometimes } \\
\text { umbonate, } \\
\text { margin } \\
\text { decurved, } \\
\text { brown, greyish } \\
\text { brown, slightly } \\
\text { yellowish- } \\
\text { brown towards } \\
\text { the margin }\end{array}$ & $\begin{array}{l}\text { Sinuate, } \\
\text { uncinateoradnate, } \\
\text { close to crowded, } \\
\text { white to } \\
\text { yellowish with } \\
\text { entire edges }\end{array}$ & $\begin{array}{l}40-170 \times 4-16 \\
\text { mm, } \\
\text { cylindrical to } \\
\text { slightly } \\
\text { attenuated } \\
\text { towards the } \\
\text { base, white to } \\
\text { pale yellow, } \\
\text { with } \\
\text { yellowish } \\
\text { longitudinal } \\
\text { striates }\end{array}$ & $\begin{array}{l}20-40 \times \\
5-10 \mu \mathrm{m}, \\
\text { clavate }\end{array}$ & $\begin{array}{l}\text { Cheilocystidia } \\
50-80 \times 8-18 \\
\mu \mathrm{m}, \text { fusiform, } \\
\text { usually with } \\
\text { crystals }\end{array}$ & $\begin{array}{l}6-9 \times 4-6 \\
\mu \mathrm{m}, \\
\text { ellipsoid, } \\
\text { ornamented } \\
\text { with } \\
\text { amyloid }\end{array}$ & $\begin{array}{l}\text { Acar et al., } \\
2017\end{array}$ \\
\hline
\end{tabular}


Table 2 (continued)

\begin{tabular}{|c|c|c|c|c|c|}
\hline \multicolumn{6}{|c|}{ M. arcuata (Bull.) Singer } \\
\hline $\begin{array}{l}50-100 \mathrm{~mm} \text {, } \\
\text { convex, then } \\
\text { plane, pale } \\
\text { brownish, } \\
\text { brownish clay } \\
\text { or coffee and } \\
\text { milk }\end{array}$ & $\begin{array}{l}\text { Emarginate, } \\
\text { deccurrent with a } \\
\text { long tooth, } \\
\text { yellowish }\end{array}$ & $\begin{array}{l}40-80 \times 7-14 \\
\mathrm{~mm}, \\
\text { fibrillose, base } \\
\text { thickened }\end{array}$ & $\begin{array}{l}\text { Cystidia } 40-60 \\
\times 10-12 \mu \mathrm{m}, \\
\text { lanseolate }\end{array}$ & $\begin{array}{l}9-10 \times 5-6 \\
\mu \mathrm{m}, \\
\text { punctate }\end{array}$ & Carleton, 1922 \\
\hline
\end{tabular}

\section{Molecular phylogeny}

ITS data matrix comprised a total of 40 sequences including 26 from NCBI. The amplified DNA fragment of the region was approximately $650 \mathrm{bp}$ long encompassing complete ITS1, 5.8S and ITS2 subregions. The data set of the region included a total of 731 positions, of which 508 were conserved, and 208 were variable (112 variable sites in ITS1, 1 in 5.8S and 95 in ITS2 subregions). Number of variations was higher in ITS1 (112) compared to ITS2 (95).

The second region, LSU, comprised 24 sequences (10 from NCBI) and yielded total lengths of 885 nucleotides with 21 nucleotide variations. In the study, we mainly used the results taken from ITS region that was more informative than LSU. The topologies of the MP and ML phylogenetic trees had no considerable differences, so only one tree (ML) was given to indicate phylogenetic relationships. Phylogenetic tree constructed based on ITS region (Figure 2) composed from two major clades which were named according to the study of Vizzini et al. (2011) and called as clade A (subgenera
Melanoleuca) and clade B (subgenera Urticocystis). Clade A and B were well supported with 99 and $98 \%$ bootstrap, respectively. Clade A consisted of four of studied species; $M$. communis, $M$. heterocystidiosa, $M$. arcuata and $M$. polioleuca. Melanoleuca arcuata from these species was placed unexpected clade. Clade B consisted of studied $M$. brevipes, $M$. dryophila, M. substrictipes, M. paedida, M. grammopodia, M. exscissa, M. angelesiana, M. graminicola, M. microcephala and $M$. melaleuca. In this clade, Melanoleuca melaleuca was placed unexpected clade. There was no discrimination at the section level in the tree.

LSU tree was not given due to less informativeness to understand phylogeny and low bootstrap values. The species could not be separated at the subgenus level (Melanoleuca and Urticocystis) and they scattered in the tree so taxonomic relations could not be resolved. Only M. paedida, $M$. substrictipes, $M$. grammopodia and $M$. exscissa evolutionarily separated from other species and caused a small cluster which was also observed in ITS tree. 


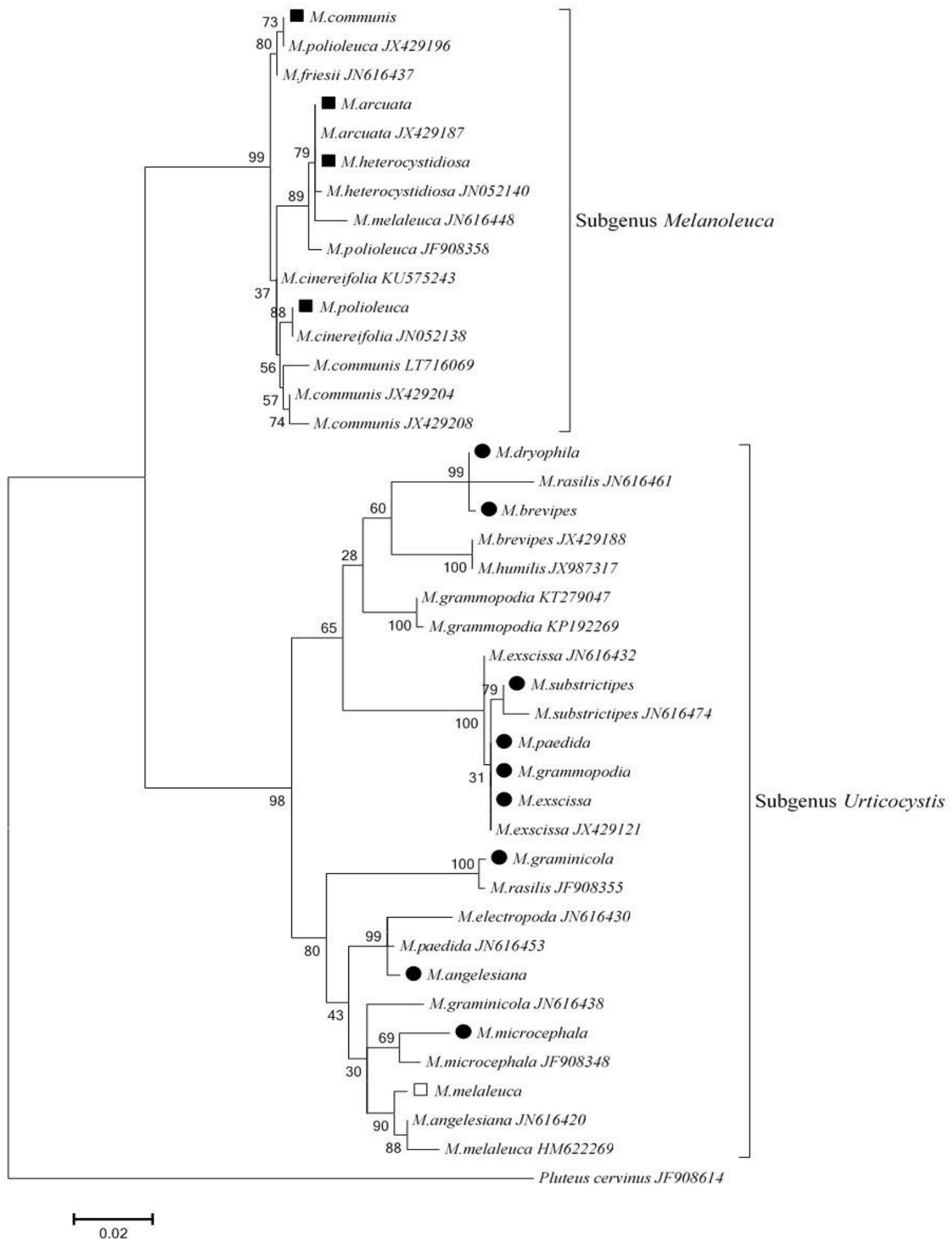

Figure 2. Maximum Likelihood phylogeny of nrITS sequences of Melanoleuca taxa. Pluteus cervinus was used as outgroup taxon. Studied species were marked with black square in Clade A and black circles in Clade B. Species whose position was incorrect from the studied species were marked with white square. Clade A; subgenus Melanoleuca. Clade B; subgenus Urticocytis. 


\section{Discussions}

Identification of Melanoleuca species is controversial due to similarities of morphological characters. Vizzini et al. (2011) indicates that environmental factors affect these characters so molecular analyses are required for delimitation of the species. For molecular analysis, one of the most important steps is to select useful region that changes according to studied genus. Therefore, two regions were used in the present study to compare and select the most useful one. Results indicated that ITS region was more useful compared to LSU to figure out phylogenetic relationships among Melanoleuca species. Genetic variation observed in the LSU was lower than that of ITS and insufficient to figure out phylogeny. In our previous study, Melanoleuca species were separated at both subgenus and section level (Acar et al., 2017) but separation was occurred at only subgenus level in the current study. The fact that number of species is increased, analysis of taxonomy is complicated.

Two major clades (Clade A and B) with high bootstrap values were observed in the constructed ITS tree. Clade A mainly included species Melanoleuca subgenus and Clade B generally composed from species Urticocystis and Acystis subgenera. Bon (1991) claimed that three subgenera are found in the genus and one of them is called as Acystis. In the present study, this subgenus was represented by only two species, $M$. graminicola and $M$. angelesiana, and they located distantly to each other. Therefore, our outcome supported the results of Vizzini and his colleagues (2011) where Acystis was rejected to be a subgenus of Melanoleuca and number of the subgenus was decreased from three to two as Melanoleuca and Urticocystis.

Clade A (subgenus Melanoleuca) consisted of species downloaded from NCBI and four of studied species; $M$. communis, $M$. heterocystidiosa, $M$. arcuata and $M$. polioleuca. This subgenus characterized by a dark-brown blackish, slate-grey pileus, whitish lamellae and mainly lageniform cystidia or rarely without cystidia (Bon, 1991; Boekhout, 1988; 1999; Vizzini et al., 2011). Sample determined as $M$. arcuata placed in this clade with $M$. arcuata (JX429187) and other species of subgenus Melanoleuca. Melanoleuca arcuata differs mainly by a very dark pileus and yellowochre lamellae only at maturity (Boekhout, 1988; Vizzini et al., 2011) so discrimination of $M$. arcuata is expected to be accurate. $M$. heterocystidia clustered with representative NCBI sample (JN052140) with $79 \%$ bootstrap value. The studied M. polioleuca and its representative retrieved from NCBI located distantly. Similar relations were seen for other species such as $M$. communis, $M$. paedida, $M$. grammopodia and $M$. graminicola. This type relationship can be originated from environmental differences. M. communis were given as new records in our previous study and detailed information (spores, basidia, cystidia etc.) can be seen (Acar et al., 2017).

Clade B (subgenus Urticocystis) consisted of studied $M$. brevipes, M. dryophila, M. substrictipes, M. paedida, M. grammopodia, M. exscissa, M. angelesiana, M. graminicola, M. microcephala, M. melaleuca and their representatives. This subgenus characterized by mainly with urticocystidia but also with macrocystidia and brightly colored pilei or lacking cystidia and absent or very rare pleurocystidia (Bon, 1991; Vizzini et al., 2011). M. angelesiana and M. graminicola species placed in this clade with $M$. paedida (JN616453), M. electropoda (JN616430) and M. rasilis (JF908355). These studied species previously were accepted to be members of subgenus Acystis (Bon, 1991) however according to Vizzini et al. (2011) Acystis is a polyphletic taxa and nests with Urticocystis. Melanoleuca angelesiana was firstly reported in Turkey with current study. It is distinguished from other species by greenishbrown stipe, amyloid warts, grey lamellae and central umbo pileus (Table 3) (Smith, 1944; Desjardin et al., 2015; Vizzini et al., 2011). 
Table 3. Comparison of Turkish specimens of Melanoleuca angelesiana and data of another authors.

\begin{tabular}{|c|c|c|c|c|c|}
\hline Pileus & Lamellae & Stipe & Basidia & Spores & $\begin{array}{l}\text { Referenc } \\
\text { es }\end{array}$ \\
\hline $\begin{array}{l}60-100 \mathrm{~mm} \text { convex, } \\
\text { smooth, central } \\
\text { umbo, dark brown }\end{array}$ & $\begin{array}{l}\text { attached to the } \\
\text { stipe, grayish }\end{array}$ & $\begin{array}{l}30-60 \times 5-15 \mathrm{~mm} \\
\text { smooth, fibrillose, } \\
\text { partial veil absent }\end{array}$ & $\begin{array}{l}23.5-37.2 \times \\
6.8-10.7 \mu \mathrm{m}\end{array}$ & $\begin{array}{l}7-10 \times 5-6.5 \\
\mu \mathrm{m}, \quad \text { elliptical, } \\
\text { amyloid, finely } \\
\text { warts }\end{array}$ & This study \\
\hline $\begin{array}{ll}50-70 \mathrm{~mm}, & \text { broad, } \\
\text { central } & \text { umbo, } \\
\text { brown } & \end{array}$ & adnate, grayish & $\begin{array}{l}50-60 \times 10-12 \\
\mathrm{~mm}, \text { fibrillose }\end{array}$ & Four spored & $\begin{array}{l}7-9 \times 4.5-6 \mu \mathrm{m} \text {, } \\
\text { elliptical, } \\
\text { amyloid warts }\end{array}$ & Smith, 1944 \\
\hline $\begin{array}{lr}20-110 & \mathrm{~mm}, \\
\text { convex, } & \text { broad, } \\
\text { sometimes } & \text { umbo, } \\
\text { dark brown } & \\
\end{array}$ & adnate, grayish & $\begin{array}{l}15-75 \times 3-15 \mathrm{~mm} \text {, } \\
\text { fibrillose }\end{array}$ & $\begin{array}{l}20-50 \times 6-13 \\
\mu \mathrm{m}\end{array}$ & $\begin{array}{l}7.5-10 \times 5-6 \\
\mu \mathrm{m}, \quad \text { elliptical, } \\
\text { verrucose, } \\
\text { amyloid warts }\end{array}$ & $\begin{array}{l}\text { Bessette et } \\
\text { al. } 1995\end{array}$ \\
\hline $\begin{array}{l}30-90 \text { mm convex, } \\
\text { low umbo, dark } \\
\text { brown, }\end{array}$ & adnate, grayish & $\begin{array}{l}30-50 \times 5-15 \mathrm{~mm} \\
\text { fibrillose, partial } \\
\text { veil absent }\end{array}$ & - & $\begin{array}{l}8-10.5 \times 5-6.5 \\
\mu \mathrm{m}, \quad \text { elliptical, } \\
\text { amyloid, finely } \\
\text { warts }\end{array}$ & $\begin{array}{l}\text { Desjardin et } \\
\text { al., } 2015\end{array}$ \\
\hline
\end{tabular}

Although M. graminicola clustered with M. rasilis (JF908355) with $100 \%$ bootstrap value morphologically they are different. Melanoleuca graminicola has not cystidia while $M$. rasilis has an urticoform cystidia (Boekhout, 1999). M. rasilis (JN616461) and M. brevipes clustered together with $99 \%$ bootstrap value in the tree. M. brevipes is differentiated from $M$. rasilis with its fusiform to lageniform cystidia and small spores covered by coarse warts. M. exscissa, $M$. grammopodia, $M$. paedida and $M$. substrictipes clustered together in both ITS and LSU (not shown) trees and morphologically they share resemble characters. They have fibrillose-smooth stipe and urticoform cystidia. However, $M$. grammopodia is characterized and separated from others by a large pileus and a long stipe (Antonin, Duriska, Jancovicova \& Tomsovsky, 2015). So, it is expected that the diagnosis is correct. M. dryophila found in Urticocystis subgenus was given as new record in our previous study and detailed information (spores, basidia, cystidia etc.) can be seen Acar et al. (2017).

Melanoleuca melaleuca is belonging to subgenus Melanoleuca but placed in subgenus Urticocystis (clade B) with $M$. melaleuca (HM622269) in the tree. Interestingly, one of M. melaleuca sample retrieved from NCBI (JN616448) placed in clade A while the other sequence (HM622269) placed in clade B with our sample $M$. melaleuca. This species is characterized by grey-brown or dark-brown pileus, amyloid warts spores and lacking cystidia (Kuo and Methven, 2014). However, morphologic characters may not enough due to homoplasy and may cause incorrect identification.

As a conclusion, morphological characters of Melanoleuca species may show high level of similarities due to convergent evolution. Therefore, only morphology may not be enough to study taxonomy of this genus. Molecular techniques are also needed to resolve some problems and selecting useful region is important. ITS region may be a proper marker to solve some taxonomic problems but it is only enough to solve problems at the subgenus level. So, additional region should be used to figure out phylogenetic relations at the species level. This study is valuable because Melaleuca specimens from Turkey have never been studied by using morphological and molecular characters. Moreover, Melanoleuca angelesiana was firstly reported for mycobiota of Turkey and detailed information about this species was also given in the paper.

\section{Acknowledgments}

The authors are grateful to Van Yüzüncü Y11 University (BAP Project No. 2017-FBY5560 ) for its financial support during the study. 


\section{Appendix 1}

Accession

downloaded from NCBI database.

species

ITS region:

Melanoleuca angelesiana (JN616420), M. brevipes (JX429188), M. humilis (JX987317), M. cinereifolia (JN052138, KU575243), M. graminicola (JN616438), $M$. melaleuca (HM622269, JN616448), M. grammopodia (KT279047, KP192269), $M$. polioleuca (JF908358, JX429196), $M$. communis (LT716069, JX429204, JX429208), M. heterocystidiosa (JN052140), M. rasilis (JN616461, JF908355), M. friesii (JN616437), M. exscissa (JX429121, JN616431), M. substrictipes (JN616474), $M$. microcephala (JF908348), M.arcuata (JX429187), M. electropoda (JN616430), M. paedida (JN616453), Pluteus cervinus (JF908614)

\section{References}

Acar, I., Dizkirici Tekpınar, A., Kalmer, A. \& Uzun, Y. (2017). Phylogenetic relationships and taxonomical positions of two new records Melanoleuca species from Hakkari province, Turkey. Biodicon 10(3), 85-93.

Altschul, S. F., Gish, W., Miller, W., Myers, E. W. \& Lipman, D.J. (1997). Basic local alignment search tool. Journal of Molecular Biology, 215, 403-410.

Antonin, V., Duriska, O., Jancovicova, S. \& Tomsovsky, M. (2015). Identity of agaricus brevipes bull. (Melanoleuca brevipes, tricholomataceae, basidiomycota). Mycological Progress 14, 107.

Bas, C., Kuyper, Th. W., Noordeloos, M. E. \& Vellinga, E.C. (1999). Flora Agaricina Neerlandica. 4th ed. CRC Press. Rotterdam, Netherlands.

Bessette, A. E., Miller, O. K., Bessette A. R. \& Miller H. H. (1995). Mushrooms of North America in color: A field guide companion to seldom-illustrated fungi. China: Syracuse UP. $172 \mathrm{pp}$.

Boekhout, T. (1988). Notulae ad floram agaricinam neerlandicam, XVI - New taxa, new combinations in Melanoleuca Pat. and notes on rare species in the Netherlands. Persoonia, 13(4), 397-431.

Boekhout, T. (1999). Melanoleuca, in: C Bas et al. (eds). Flora Agaricina Neerlandica 4.A.A. Balkema, Rotterdam. 153-165.

Bon, M. (1991). Flore mycologiqued'Europe, 2 Les tricholomesetressemblants. Revue de Mycologie Mémóire Hors-Série, 2, 163.
Breitenbach, J. \& Kränzlin, F. (1991). Fungi of Switzerland. Verlag Mykologia, 3rd ed. Lucerne, Switzerland.

Bresinsky, V.A. \& Stangl, J. (1977). Beiträgezur revision $\mathrm{m}$. britzelmayrs hymenomycetenaus südbayern. Zeitsehr.f.Pilzkunde, (43) ,145173.

Brown, P.S., Rigdon-Huss, A.R. \& Jumpponen, A. (2014). Analyses of ITS and LSU gene regions provide congruent results on fungal community responses. Fungal Ecology, (9), 65-68.

Buczacki, S. (2012). Collins fungi guide: the most complete field guide to the mushrooms and toadstools of Britain \& Ireland. London, UK: Harper Collins Publishers.

Carleton, R. (1922). British basidiomycetae, Cambridge University Press, UK.

Clémençon, H. (2009). Methods for working with macrofungi: laboratory cultivation and preparation of larger fungi for light microscopy. Berchtesgaden: Berchtesgadener Anzeiger.

Dähncke, M. R. (2004). 1200 Pilze in farbfotos. Switzerland: ATVerlag Aarau.

Desjardin, D. E., Wood, M. G. \& Stevens, F. A. (2015). California mushrooms: the comprehensive identification guide. Portland, OR.: Timber Press. 560.

Doyle, J. J. \& Doyle, J. L. (1987). A rapid DNA isolation procedure for small quantities of fresh leaf tissue. Phytochemical Bulletin, (19), 11-15.

Duriska, O., Antonin, V., Para, R., Tomsovsky, M. \& Jancovicova, S. (2017). Taxonomy, ecology and distribution of melanoleuca strictipes (basidiomycota, agaricales) in Europe. Czech Mycology, 69(1), 15-30.

Felsenstein, J. (1985). Confidence limits on phylogenies: An approach using the bootstrap. Evolution, (39), 783-791.

Garcia, M. S., Blanco, J. C. \& Matheny, P. B. (2013). Taxonomic revision of the genus Melanoleuca in Mexico and description of new species. Revista Mexicana de Biodiversidad, 111-127.

Gerault, A. (2005). Florule evolutive des basidiomycotina du finistere heterobasidiomycetes tricholomatales. Version 2.1. France.

Hinrikson, H. P., Hurst, S. F., Lott, T. J., Warnock, D. W. \& Morrison, C. J. (2005). Assessment of ribosomal large-subunit D1D2, internal transcribed spacer 1, and internal transcribed spacer 2 regions as targets for molecular identification of medically important aspergillus species. Journal of Clinical Microbiology, (43), 2092-2103.

Hyde, K. D., Udayanga, D., Manamgoda, D. S., Tedersoo, L., Larsson, E., Abarenkov, K., 
Bertrand, Y. J. K., Oxelman, B., Hartmann, M., Kauserud, H., Ryberg, M., Kristiansson, E. \& Nilsson, R. H. (2013). Incorporating molecular data in fungal systematics: a guide for aspiring researchers. Current Research in Environmental \& Applied Mycology, 3(1), 132.

Jordan, M. (2004). The Encyclopedia of fungi of Britain and Europe. UK.: Frances Lincoln Publishers P. 51.

Kibby, G. (2016). Melanoleuca castaneofusca new to Britain. Field Mycology, 17(3):95-97.

Kuo, M. \& Methven, S. A. (2014). Mushrooms of the Midwest. Urbana, Chicago and Springfield: University of Illinois Press.

Murrill, W. A. (1913). The agaricaceae of the Pacific Coast - IV. New species of Clitocybe and Melanoleuca. Mycologia, (5), 206-223.

Mycobank database. (2018). Melanoleuca. http://www.mycobank.org/BioloMICS.aspx?T able $=$ Mycobank $\&$ Rec $=56901 \&$ Fields $=$ All, Westerdijk Fungal Biodiversity Institue, München, Germany. (Accessed: 25.02.2018)

Phillips, R. (2006). Mushrooms. Published August, (18th ed.) by Pan MacMillan.

Schoch, C. L., Seifert, K. A., Huhndorf, S., Robert, V., Spouge, J. L., Levesque, C. A. \& Chen, W. (2012). Fungal Barcoding Consortium Nuclear ribosomal internal transcribed spacer (ITS) region as a universal DNA barcode marker for Fungi. Proceedings of the National Academy of Sciences, 109, 6241-6246.

Sesli, E. \& Denchev, C.M. (2014). Checklists of the myxomycetes, larger ascomycetes, and larger basidiomycetes in Turkey. Mycotaxon Checklists Online, 1-136.

Singer R. (1986). The Agaricales in Modern Taxonomy. (4th ed.) Koenigstein Königstein im Taunus, Germany: Koeltz Scientific Books.

Smith, A. H. (1944). New North American Agarics. Mycologia, 36, 242-262.

Solak, M.H., Işıloğlu, M., Erbil, K. \& Allı, H. (2015). Macrofungi of Turkey checklist. (2nd ed.) İzmir: Üniversiteliler Ofset.

Tamura, K. \& Nei, M. (1993). Estimation of the number of nucleotide substitutions in the control region of mitochondrial DNA in humans and chimpanzees. Molecular Biology and Evolution, (10), 512-526.

Tamura, K., Stecher, G., Peterson, D., Filipski, A., M. \& Kumar, S. (2013). MEGA 6: Molecular evolutionary genetics analysis Version 6.0. Molecular Biology Evolution, 30(12), 2725-2729.

Thompson, J. D., Higgins, D. G. \& Gibson, T. J. (1994). Clustal W improving the sensitivity of progressive multiple sequence alignment through sequence weighting, position-specific gap penalties and weight matrix choice. Nucleic Acids Research, (22), 4673-4680.

Undan, J. Q., Alfonso, D. O., Dulay, R. M., Leon, A. M., Kalaw, S. P. Undan J. R. \& Reyes, R. G. (2016). Molecular identification and phylogeny of different macrofungi in Mt. Bangkay, Cuyapo, Nueva Ecija, Philippines based on ITS nrDNA region. Advances in Environmental Biology, 10(12), 35-42

Vesterholt, J. \& Knudsen, H. (2008). Funga Nordica-Agaricoid, boletoid and cyphelloid genera. Nordsvamp, Copenhagen.

Vilgalys, R. \& Hester, M. (1990). Rapid genetic identification and mapping of enzymatically amplified ribosomal DNA from several Cryptococcus species. Journal of Bacteriology 172, 4239-4246.

Vizzini, A., Para, R., Fontenla, R., Ghignone, S. \& Ercole, E. (2011). A preliminary ITS phylogeny of Melanoleuca (Agaricales) with special reference to European taxa. Mycotaxon, 118, 361-381.

Wen, J. \& Zimmer, E. A. (1996). Phylogeny and Biogeography of Panax L. (the Ginseng Genus, Araliaceae): Inferences from ITS Sequences of Nuclear Ribosomal DNA. Molecular Phylogenetics and Evolution 6, 167-177.

Yıldız, S., Gürgen, A. \& Can, Z. (2017). In vitro bioactive properties of some wild mushrooms collected from Kastamonu province. Kastamonu University Journal of Forestry Faculty, 17(3), 523-530.

Yu, X. D., Lv, S. X., Ma, D., Li, F. F., Lin, Y. \& Zhang, L. (2014). Two new species of Melanoleuca (Agaricales, Basidiomycota) from northeastern China, supported by morphological and molecular data. Mycoscience, 55, 456-461. 